\title{
New evolutionary models for pre-main sequence and main sequence low-mass stars down to the hydrogen-burning limit
}

\author{
Isabelle Baraffe ${ }^{1,2}$, Derek Homeier ${ }^{2}$, France Allard ${ }^{2}$, and Gilles Chabrier ${ }^{2,1}$ \\ 1 University of Exeter, Physics and Astronomy, EX4 4QL Exeter, UK \\ e-mail: i. baraffe@ex.ac.uk \\ 2 École Normale Supérieure, Lyon, CRAL (UMR CNRS 5574), Université de Lyon, 69364 Lyon Cedex 07, France \\ e-mail: [derek .homeier; fallard; chabrier] @ens-lyon.fr
}

Received 8 December 2014 / Accepted 24 February 2015

ABSTRACT

\begin{abstract}
We present new models for low-mass stars down to the hydrogen-burning limit that consistently couple atmosphere and interior structures, thereby superseding the widely used BCAH98 models. The new models include updated molecular linelists and solar abundances, as well as atmospheric convection parameters calibrated on 2D/3D radiative hydrodynamics simulations. Comparison of these models with observations in various colour-magnitude diagrams for various ages shows significant improvement over previous generations of models. The new models can solve flaws that are present in the previous ones, such as the prediction of optical colours that are too blue compared to $\mathrm{M}$ dwarf observations. They can also reproduce the four components of the young quadruple system LkCa 3 in a colour-magnitude diagram with one single isochrone, in contrast to any presently existing model. In this paper we also highlight the need for consistency when comparing models and observations, with the necessity of using evolutionary models and colours based on the same atmospheric structures.
\end{abstract}

Key words. stars: evolution - stars: low-mass - stars: pre-main sequence - Hertzsprung-Russell and C-M diagrams - convection

\section{Introduction}

In 1998, our team released a set of evolutionary models for low-mass stars (Baraffe et al. 1998, hereafter BCAH98) based on the so-called NextGen atmosphere models (Hauschildt et al. 1999) that marked a new era of models that consistently coupled interior and atmosphere structures. These models became very popular because they successfully reproduce various observational constraints, such as mass-luminosity and mass-radius relationships and colour-magnitude diagrams. The models, however, had some important shortcomings, such as predicting optical $(V-I)$ colours that are too blue for a given magnitude (see Sect. 3.1). Later generations of models included improved molecular linelists for various atmospheric absorbers, such as the AMES linelists used in the Dusty and Cond models (Chabrier et al. 2000; Allard et al. 2001; Baraffe et al. 2003), but they still show shortcomings (see Sect. 3.2). After a long effort to solve these flaws, efforts have paid off with the release of current models that supersede the BCAH98 models. In this paper, we describe the main physical ingredients of the models and compare them to a selection of observations that highlight the improvement of these new models over previous ones.

\section{Model description}

Evolutionary calculations are based on the same input physics describing stellar and substellar interior structures as are used in Chabrier \& Baraffe (1997) and Baraffe et al. (1998). The major changes concern the atmosphere models, which provide the outer boundary conditions for the interior structure calculation, and the colours and magnitudes for a given star mass at any given age. Substantial changes have been made since the NextGen atmosphere models used in the BCAH98 evolutionary models. A preliminary set of atmosphere models, referred to as the BT-Settl models (Allard et al. 2012a,b; Rajpurohit et al. 2013), include some of these changes, which are briefly summarised below. More recent modifications concerning the treatment of convection are described in Sect. 2.3.

\subsection{Molecular linelists and cloud formation}

Line opacities for several important molecules have been updated, notably the water linelist from Barber et al. (2006), metal hydrides such as $\mathrm{CaH}, \mathrm{FeH}, \mathrm{CrH}$, TiH from Bernath (2006), vanadium oxide from Plez (2004, priv. comm.), and carbon dioxide from Tashkun et al. (2004). For TiO, the present set of atmosphere models uses the linelist from Plez (1998). This list is not as complete at high energies as the AMES linelist (Schwenke 1998) adopted in Allard et al. (2001, 2012a), with only $11 \times$ $10^{6}$ lines compared to the $160 \times 10^{6}$ of Schwenke (1998). But the Plez linelist reproduces the overall band strengths better and thus generally improves the optical colours (Fig. 4). Obviously, the field is still in need of a new, complete, and accurate theoretical $\mathrm{TiO}$ linelist to allow quantitative high-resolution spectroscopic analysis of this important molecule, as recently pointed by the high-resolution transmission spectrum analysis of a transiting exoplanet (Hoeijmakers et al. 2015).

Condensation of up to 200 types of liquids and solids is included in the atmospheric equation of state. The formation and sedimentation of clouds and depletion of condensible material is treated in the self-consistent timescale approach of 
Allard et al. (2012a). They used a monodisperse grain size distribution for each cloud layer, but current version of the models now considers a log-normal distribution over 1.8 decades in size in 12 bins with the altitude-dependent size determined by the same timescale formalism as in Allard et al. (2012a). The set of 60 grain types included in calculating the cloud opacity has also been updated with the optical data for several low-temperature condensates, which become important in the atmospheres of $\mathrm{T}$ dwarfs, which are not discussed in this paper (Homeier et al., in prep.).

\subsection{Solar abundances}

Solar abundances have been revised over the past decade based on radiation hydrodynamical simulations of the solar photosphere combined with 3D non-local thermodynamical equilibrium radiative transfer (Asplund et al. 2009; Caffau et al. 2011). This leads to a substantial reduction of carbon, nitrogen, and oxygen abundances compared to the abundances of Grevesse et al. (1993), previously used in the NextGen and AMESDusty/Cond models. The present models adopt the solar composition of Asplund et al. (2009) with revisions of the elemental abundances of C, N, O, Ne, P, S, K, Fe, Eu, Hf, Os, and Th obtained by the CIFIST project (Caffau et al. 2011). This yields, in particular, a slight upward revision of carbon, nitrogen, and oxygen abundances and an increased total heavy element fraction by mass, with $Z=0.0153$ compared to 0.0122 in Asplund et al. (2005) and 0.0134 in Asplund et al. (2009). Although the individual differences with the Asplund et al. (2009) elemental abundances are modest, Antia \& Basu (2011) and Basu \& Antia (2013) have found that the higher overall metal content of the CIFIST abundances is in better agreement with helioseismology results for the solar interior. The helium abundance adopted in the atmosphere models is fixed to $Y_{\text {ini }}=0.271$, which is representative of the initial solar helium abundance (Serenelli \& Basu 2010; Basu \& Antia 2013).

\subsection{Treatment of convection}

Radiative-convective equilibrium in the PHOENIX atmosphere models is calculated using the mixing length theory (or MLT, see Kippenhahn \& Weigert 1990). The main free parameter within this framework is the mixing length $l_{\mathrm{mix}}$, expressed in terms of the pressure scale height $H_{\mathrm{P}}$ and $l_{\text {mix }}$ describes the efficiency of the convective energy transport in terms of the superadiabaticity of the temperature gradient. All previous PHOENIX models have used a constant value for $l_{\text {mix }} \equiv l_{\text {mix }}^{\mathrm{atm}}: l_{\text {mix }}^{\mathrm{atm}}=H_{\mathrm{P}}$ in the NextGen and Cond/Dusty models and $l_{\text {mix }}^{\text {atm }}=2 \times H_{\mathrm{P}}$ in Allard et al. (2012a).

In the present work, the determination of $l_{\text {mix }}^{\text {atm }}$ is inspired by the work of Ludwig et al. (1999, 2002). It is based on comparisons between 1D MLT models and 2D/3D RHD simulations that cover main sequence and pre-MS models down to the hydrogen-burning limit and below (Freytag et al. 2010, 2012). Since the RHD models include a basic treatment of dust formation, they also allow the calibration of late M dwarfs, where cloud opacity is becoming relevant. This calibration yields a value of $l_{\text {mix }}^{\text {atm }} \approx 1.6 \times H_{\mathrm{P}}$ for the Sun. The value of $l_{\text {mix }}^{\text {atm }}$ increases for later type stars, up to values $\gtrsim 2 \times H_{\mathrm{P}}$ for the coolest and densest models ( $T_{\text {eff }}<3000 \mathrm{~K}$ and $\log g>4.5$ ). Comparisons between 1D and RHD pressure-temperature profiles are shown in Fig. 1. The adopted value of $l_{\mathrm{mix}}^{\mathrm{atm}}$, for a given $T_{\mathrm{eff}}$ and $\log g$, provides the best overall agreement that can be obtained; however,
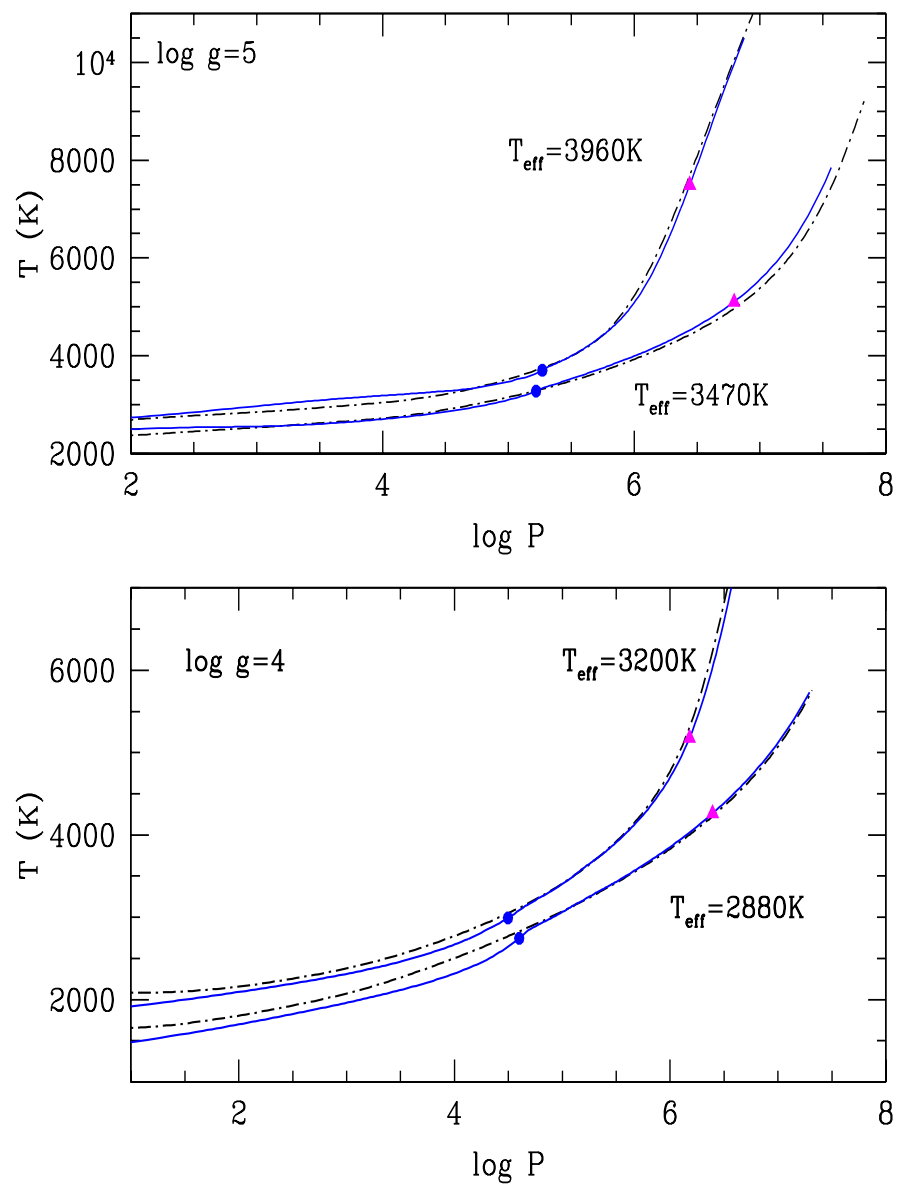

Fig. 1. Comparisons of pressure-temperature atmospheric profiles between present 1D (solid blue) and $\mathrm{CO}^{5}$ BOLD RHD (dash-dot black) models, for different effective temperatures and surface gravities, as indicated in the panels for each curves. Pressure unit is dyne $/ \mathrm{cm}^{-2}$. Calibration of $l_{\text {mix }}$ based on such comparisons yields $l_{\text {mix }}^{\text {atm }}=1.64 \times H_{\mathrm{P}}$ for $T_{\text {eff }}=2880, \log g=4.0 ; l_{\text {mix }}^{\mathrm{atm}}=1.58 \times H_{\mathrm{P}}$ for $T_{\text {eff }}=3200, \log g=4.0$; $l_{\text {mix }}^{\text {tat }}=1.69 \times H_{\mathrm{P}}$ for $T_{\text {eff }}=3470, \log g=5.0 ; l_{\text {mix }}^{\text {atm }}=1.58 \times H_{\mathrm{P}}$ for $T_{\text {eff }}=3960, \log g=5.0$. The dot on each solid curve indicates the onset of convection and the triangle indicates the location of optical depth $\tau=100$ (Chabrier \& Baraffe 1997) where the transition to the interior model is performed.

the agreement is not perfect all the way from the top to the bottom of the atmosphere, reflecting limitations in the MLT formalism for properly handling atmospheric convection (Homeier et al., in prep.).

\subsection{Evolutionary models}

Current models cover the evolution of pre-MS and MS stars from $0.07 M_{\odot}$ to $1.4 M_{\odot}$. Calibration of a $1 M_{\odot}$ star sequence to fit the fundamental parameters of the $\operatorname{Sun}\left(R_{\odot}, L_{\odot}\right)$ at its age requires a value for the interior structure of the mixing length $l_{\text {mix }} \equiv l_{\text {mix }}^{\text {int }}=1.6 \times H_{\mathrm{P}}$ and a helium abundance $Y=0.28$. We used this value of helium for the complete grid of evolutionary models. The value adopted in the atmosphere models is slightly different ( $Y=0.271$, see Sect. 2.2). To appreciate the effect of this inconsistency, we have recalculated a full grid of evolutionary models assuming the same He abundance $Y=0.271$ in the interior structure as in the atmosphere models. The effect of such a variation in $Y$ on $T_{\text {eff }}$ for the whole range of masses considered is less than $1 \%$. The maximum effect on the luminosity is found 
I. Baraffe et al.: New evolutionary models for pre-main sequence and main sequence low-mass stars

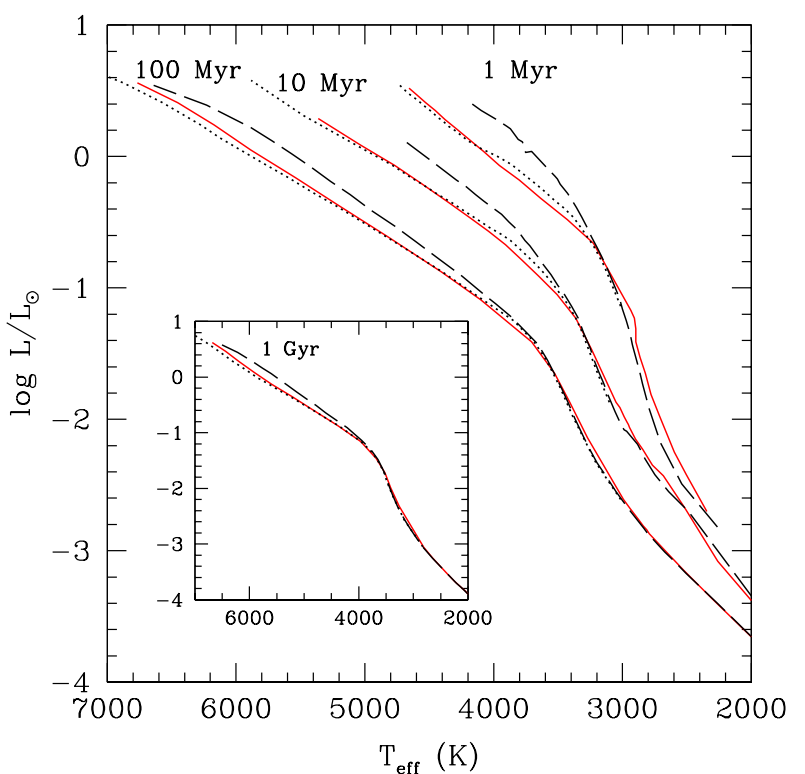

Fig. 2. Comparison of present models with the BCAH98 models for various isochrones, as indicated in the figure and inset. Solid (red): present models; long dash (black): BCAH98 models with $l_{\text {mix }}^{\text {int }}=H_{\mathrm{P}}$; dotted (black): BCAH98 with $l_{\text {mix }}^{\text {int }}=1.9 \times H_{\mathrm{P}}$.

for the highest masses $\left(M \gtrsim 1 M_{\odot}\right)$ and reaches at most $10 \%$. For a $1 M_{\odot}$ star, the effect on the luminosity is less than $5 \%$ for ages $\$ 2 \mathrm{Gyr}$, and up to $10 \%$ for ages $\gtrsim 2$ Gyr. We have also computed a few atmosphere models again with $Y=0.28$ for a range of $T_{\text {eff }}$ between $2600 \mathrm{~K}$ and $6000 \mathrm{~K}$ and of gravities $\log g$ between 3.5 and 5. The effect of such a variation in $Y$ is negligible on the atmospheric thermal profiles (less than a $1 \%$ effect on the pressure at a given temperature) and on the photometry (differences less than 0.01 mag for all colours explored).

We fixed $l_{\text {mix }}^{\text {int }}=1.6 \times H_{\mathrm{P}}$ for the complete grid of evolutionary models for the interior structure. A fully consistent approach would be to adopt the value obtained for the corresponding atmosphere model, i.e $l_{\text {mix }}^{\text {int }}=l_{\text {mix }}^{\text {atm }}$. We checked that this is unnecessary. Indeed, values of $l_{\text {mix }}^{\text {atm }}$ only start to depart significantly from $1.6 \times H_{\mathrm{P}}$ for the coolest and densest models $\left(T_{\mathrm{eff}}<3000 \mathrm{~K}\right.$ and $\log g>4.5)$, reaching values up to $2 \times H_{\mathrm{P}}$ or more. This concerns masses $<0.2 M_{\odot}$, where a variation of $l_{\text {mix }}^{\text {int }}$ between $1.6 \times H_{\mathrm{P}}$ and $2 \times H_{\mathrm{P}}$ has an insignificant effect on the evolution.

As illustrated in Fig. 2, the new models predict different positions of isochrones than do the BCAH98 models. Below $T_{\text {eff }} \lesssim 2800 \mathrm{~K}$, dust starts to form in the upper atmosphere and affects atmospheric thermal profiles and spectra. In the current grid of evolutionary models with $T_{\text {eff }}>2000 \mathrm{~K}$, the effects of cloud opacity only gradually become visible for the latest $\mathrm{M}$ and early L dwarfs ( $T_{\text {eff }} \lesssim 2500 \mathrm{~K}$ ). They are becoming crucial for models of $\mathrm{L}$ and $\mathrm{T}$ dwarfs, which will be examined in a forthcoming study.

Theoretical mass-radius relationships for $0.1 \mathrm{Gyr}$ and $1 \mathrm{Gyr}$ are shown in Fig. 3 for different sets of models. As shown in Chabrier \& Baraffe (1997), the mass-radius relationship for lowmass stars is essentially fixed by the equation of state, with a very small dependence on the atmosphere treatment. The differences between present models and the BCAH98 models essentially stem from the different values of the interior mixing length $l_{\text {mix }}^{\text {int }}$ used. As shown in Fig. 3, the effect of the interior He abundance $Y_{\text {int }}$ slightly affects the mass-radius relationship for masses $\gtrsim 1 M_{\odot}$.

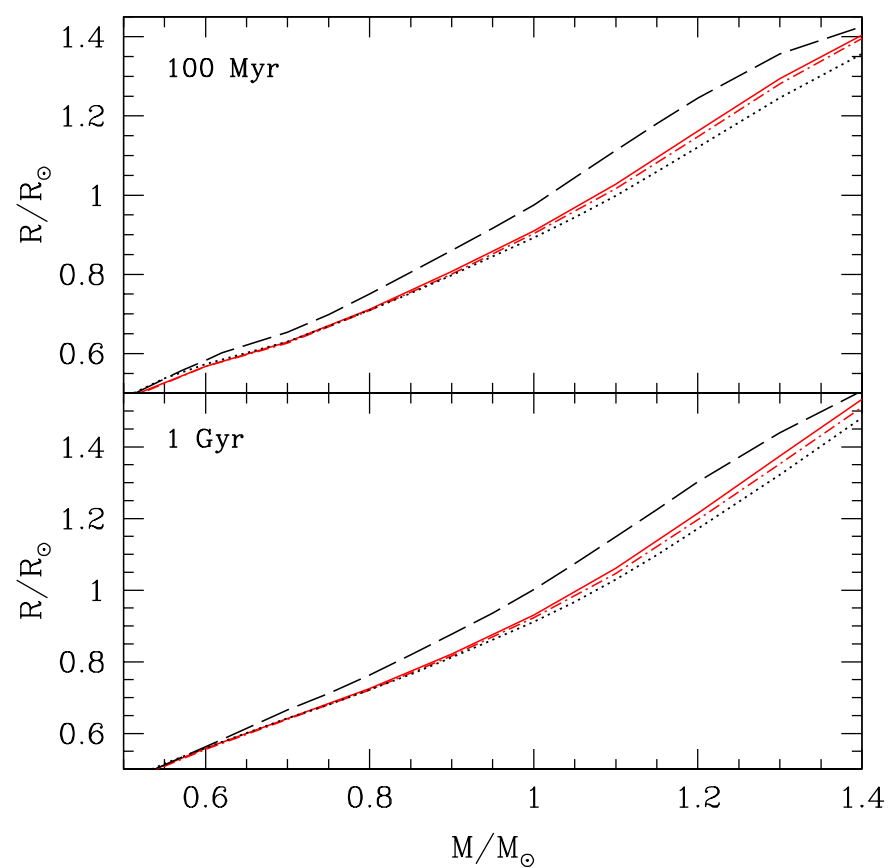

Fig. 3. Comparison of theoretical mass-radius relationships for ages of $0.1 \mathrm{Gyr}$ (upper panel) and $1 \mathrm{Gyr}$ (lower panel) and based on different sets of models: present models with $Y_{\text {int }}=0.28$ (solid red) and $Y_{\text {int }}=$ 0.271 (short-dash red); BCAH98 models with $l_{\text {mix }}^{\text {int }}=H_{\mathrm{P}}$ (long-dash black) and $l_{\text {mix }}^{\text {int }}=1.9 \times H_{\mathrm{P}}($ dot black $)$.

\section{Comparison with observations}

In this section, we highlight the significant improvement provided by this new set of models, which solve some of the problems of the BCAH98 models, through a selection of observational tests. Colour-magnitude diagrams (CMDs) provide the best validity test, as they test the consistency between interior and atmosphere structures.

\subsection{Optical colours}

As mentioned in the introduction, a well-known flaw of the BCAH98 models is the prediction of colours that are too blue $(V-I)$. Figure 4 compares models at $1 \mathrm{Gyr}$ with solarneighbourhood and disk objects with parallax (Monet et al. 1992; Leggett 1992; Cantrell et al. 2013). The comparison shows that this persistent problem of the models seems to be solved by several improvements. The most important one is the use of the TiO linelist from Plez (1998). The impact of the TiO linelist has already been noticed with the generation of models following the NextGen ones, such as the Dusty models (Chabrier et al. 2000; Allard et al. 2001). The former ones use the linelist by Jorgensen (1994), while the latter ones include the AMES TiO linelist of Schwenke (1998). The improvement in $(V-I)$ colours of models that include the AMES TiO linelist of Schwenke (1998) was significant (see Fig. 4), but still unable to yield satisfactory agreement with observations. Indeed, an offset in $(V-I)$ colours of $\sim 0.4 \mathrm{mag}$ remained between $T_{\text {eff }} \sim 3600 \mathrm{~K}\left(M_{\mathrm{V}} \sim 10\right)$ and $T_{\text {eff }} \sim 2300 \mathrm{~K}\left(M_{\mathrm{V}} \sim 19\right.$, see Chabrier et al. 2000). Improvement due to the implementation of the Plez (1998) $\mathrm{TiO}$ linelist in the new models is illustrated in Fig. 4 by the comparison between present models (TiOPl) and the same models based on the AMES TiO linelist (TiOAM). 


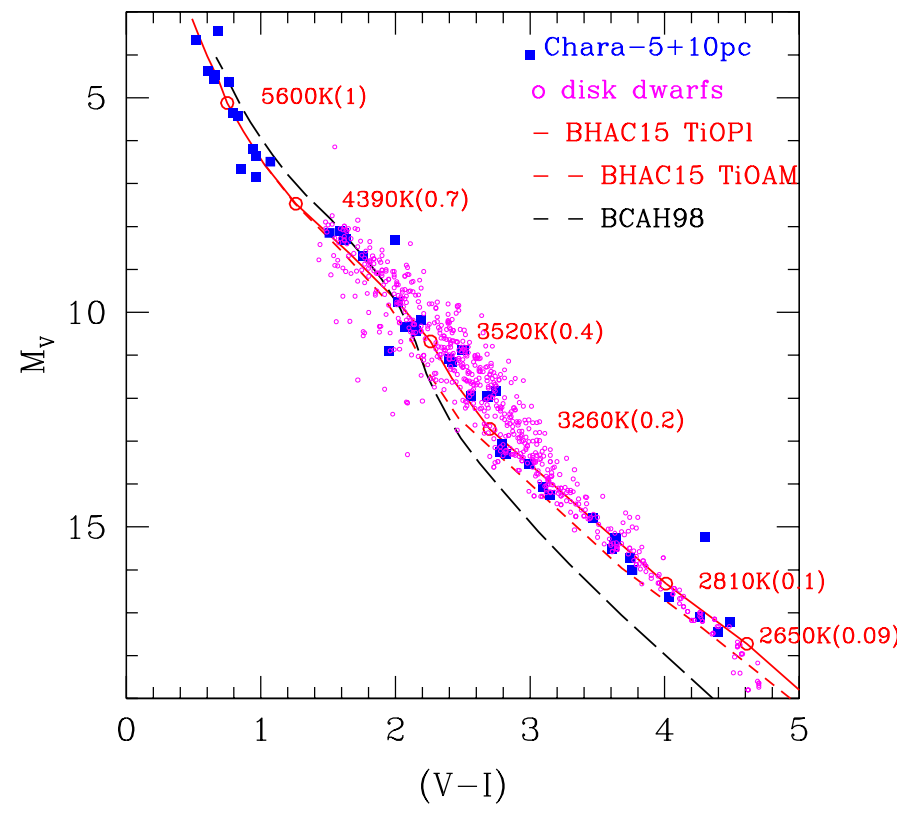

Fig. 4. Comparison of $1 \mathrm{Gyr}$ isochrones with disk dwarfs in an optical CMD. Present models based on the Plez TiO (solid red) and the AMES TiO (dash red) linelists are shown, along with the BCAH98 models (long dash black). The magenta open circles are disk objects with parallax (Monet et al. 1992; Leggett 1992) and the blue squares are from the recent $5 \mathrm{pc}$ and $10 \mathrm{pc}$ solar neighbourhood samples of Cantrell et al. (2013) with more accurate parallaxes and cleaned of binary systems. The numbers close to the red open circles on the solid curve give $T_{\text {eff }}$ and mass (in $M_{\odot}$ in the brackets) for selected models.

Another positive effect stems from the change in solar abundances, with a decrease in oxygen abundance by $\sim 22 \%$ between the Grevesse et al. (1993), used in previous sets of models, and the presently used Caffau et al. (2011) abundances. As a consequence of relatively less $\mathrm{O}$, the flux in the IR generally increases owing to weaker water absorption. Because of flux redistribution, the flux in the $V$-band decreases relative to the flux at longer wavelengths. This is an important effect, illustrating how water abundance affects optical colours due to flux redistribution.

\subsection{Near-infrared colours}

The new models yield also a significantly better match with observations for relatively old objects (age $\gtrsim 1 \mathrm{Gyr}, \log g \gtrsim 4.5$ ) in near-IR CMDs, as illustrated in Fig. 5. This stems from the use of the more complete and accurate water linelist of Barber et al. (2006), compared to previously used ones, namely Miller et al. (1994) in NextGen and Partridge \& Schwenke (1997) in Dusty/Cond.

To illustrate the impact of the new models at low gravities and in a similar range of effective temperatures $\left(T_{\text {eff }}>2000 \mathrm{~K}\right)$, we also compared them to data in the young cluster $\sigma$ Orionis (Peña Ramírez et al. 2012), with an age of a few Myr (log $g \lesssim 4)$. The results are shown in Fig. 6 in various CMDs. Fluxes from the models have been transformed in the VISTA filters to be consistent with the data of Peña Ramírez et al. (2012). Comparison of models in CMDs (or luminosity- $T_{\text {eff }}$ diagrams) with observations in young clusters and star formation regions should always be taken with caution owing to the observed luminosity spread and the effect of initial conditions and accretion history (Baraffe et al. 2002, 2009, 2012). The comparison in Fig. 6, however, is illustrative of the overall improvement in ZJHK filters of the new
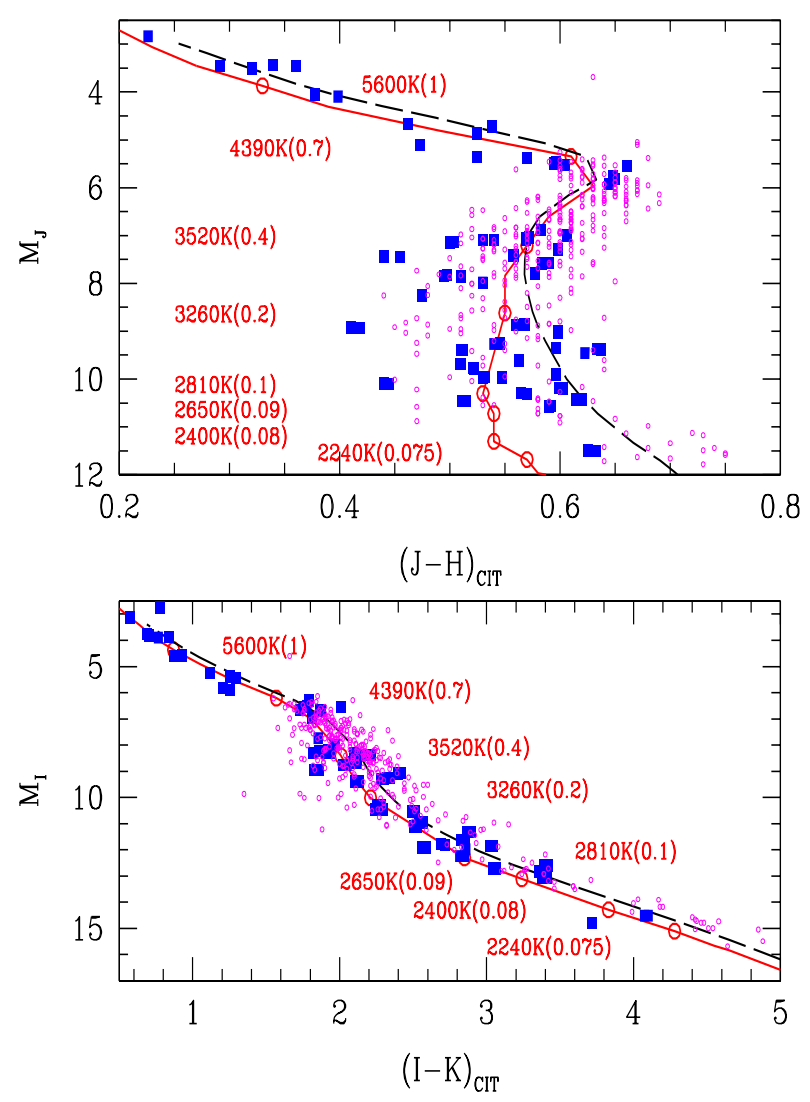

Fig. 5. Comparison of $1 \mathrm{Gyr}$ isochrones with disk dwarfs in nearIR CMDs in CIT filters. Solid (red): new models; long dash (black): BCAH98 models with $l_{\text {mix }}^{\text {int }}=H_{\mathrm{P}}$. Symbols are the same as in Fig. 4 .

models compared to the BCAH98 ones. The latter indeed provide poor agreement with observations at young ages in near-IR CMDs, as illustrated in Fig. 6. For this reason, models had to be compared with data in $L-T_{\text {eff }}$ or HR diagrams, which implied the use of very uncertain bolometric corrections and spectral type$T_{\text {eff }}$ or colour- $T_{\text {eff }}$ scales. The agreement of the new models with observations, however, deteriorates at the faintest magnitudes by up to $\sim 0.1 \mathrm{mag}$ in $(J-H)$ and $\sim 0.4 \mathrm{mag}$ in $(J-K)$. Given photometric uncertainties and possible $K$-band excess at this age owing to the presence of a circumstellar disk, it is premature to draw any conclusion on the reason for these discrepancies.

\subsection{Multiple systems}

Stringent tests of evolutionary models are provided by coeval multiple systems. The recent discovery of the quadruple premain sequence system $\mathrm{LkCa} 3$ is interesting in this respect (Torres et al. 2013). If one admits that the four components of LkCa 3 should be coeval and must lie on the same isochrone, they make an excellent test of evolutionary models. This supposes that early history of accretion has not altered their structure (Baraffe et al. 2009, 2012) and that their position in a CMD or $L-T_{\text {eff }}$ diagram can be reproduced by non-accreting evolutionary models.

With this assumption, Torres et al. (2013) find that no currently available model is able to reproduce the position of the four components with one single isochrone in a $(V-H)-M_{V}$ CMD. In contrast, our new models do fulfil this stringent constraint within the error bars (see Fig. 7). The new models agree with observations for an age of 1.6 Myr. In comparison, the 
I. Baraffe et al.: New evolutionary models for pre-main sequence and main sequence low-mass stars

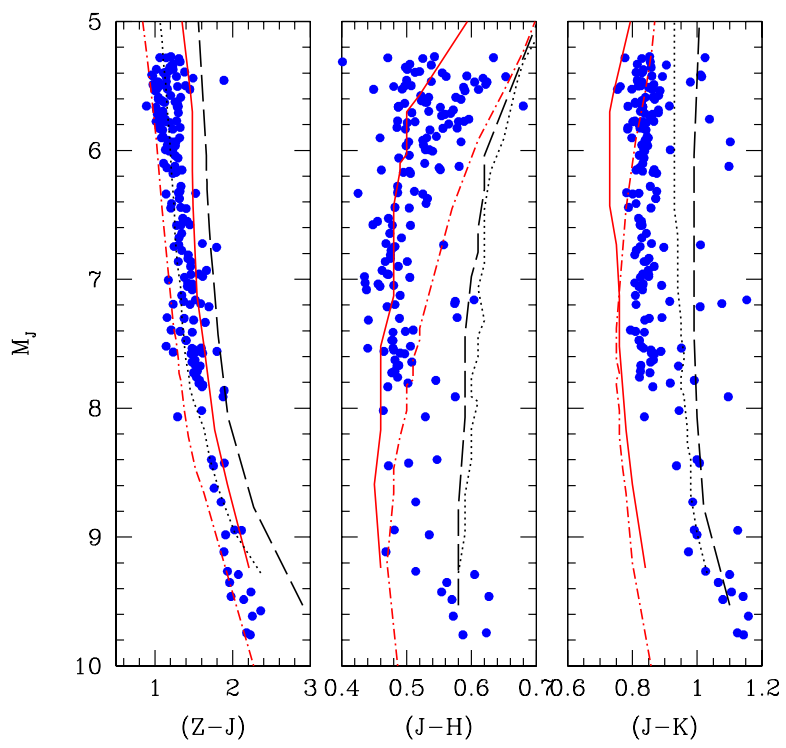

Fig. 6. Comparison of models with observations in $\sigma$ Orionis in various CMDs (VISTA filters). Isochrones of $1 \mathrm{Myr}$ and $10 \mathrm{Myr}$ are displayed for various sets of models: present models (red): solid (1 Myr) and dash$\operatorname{dot}(10 \mathrm{Myr})$. BCAH98 models with $l_{\text {mix }}^{\text {int }}=H_{\mathrm{P}}$ (black): long dashed (1 Myr) and dot (10 Myr). The data (blue dots) are from Peña Ramírez et al. (2012) using a distance modulus of 7.73 (i.e. a distance of $352 \mathrm{pc}$ ). The hydrogen-burning limit $\left(M=0.07 M_{\odot}\right)$ is located at $M_{J} \sim 6\left(T_{\text {eff }} \sim\right.$ $2900 \mathrm{~K}, \log g \sim 3.5)$ at $1 \mathrm{Myr}$ and $M_{\mathrm{J}} \sim 8\left(T_{\mathrm{eff}} \sim 2950 \mathrm{~K}, \log g \sim 4.2\right)$ at $10 \mathrm{Myr}$.

best-fit 6.3 Myr isochrone of the BCAH98 models with $l_{\text {mix }}^{\text {int }}=$ $H_{\mathrm{P}}$ is unable to match the coolest component $\mathrm{LkCa} 3 \mathrm{Ab}$, which can be fitted by an isochrone of 2.5 Myr. A similar problem arises with the BCAH98 models using $l_{\text {mix }}^{\text {int }}=1.9 \times H_{\mathrm{P}}$. The same failure was reported by Torres et al. (2013, see their Fig. 6) with the Dartmouth models (Dotter et al. 2008).

As a final note, even if accretion history can have an impact on the structure of young objects, as suggested by Baraffe et al. (2012), this does not necessarily affect all objects in the same way. Even for binary or multiple systems, each component has its own accretion history, and its structure may be differently altered by accretion, since the effect depends on the total amount of accreted mass (see Baraffe et al. 2012 for details). Disentangling the remaining uncertainties of models and possible effects of accretion when comparing models to young multiple systems is thus not straightforward. We hope that many more such multiple systems, spanning a wide range of masses and ages, will be discovered in the near future to provide more clues to the remaining model uncertainties (e.g. convection treatment, opacity uncertainty) and/or to the impact of accretion for the youngest systems.

\subsection{Tests of stellar evolutionary models}

For the sake of testing evolutionary models against observations, it is crucial to use the synthetic colours and magnitudes predicted by the same atmosphere models as those that provide the outer boundary conditions for the interior structure. Using "hybrid" models, based on atmospheric thermal profiles for the interior structure from one set of atmosphere models and colours/magnitudes from another set, yields incorrect results. Figure 8 illustrates this point, showing in two CMDs for various isochrones a comparison between the present set of models, the BCAH98 models, and a hybrid model based on BCAH98

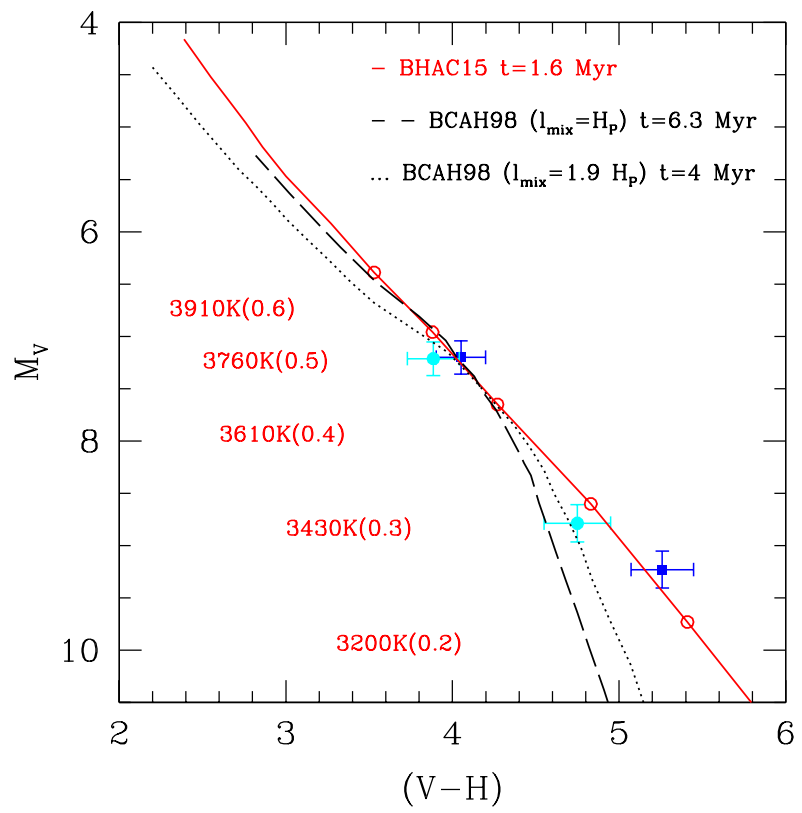

Fig. 7. Comparison of models with the four components of the quadruple system LkCa 3 (Torres et al. 2013). The best-fit isochrone is shown for various sets of models. Present models for an age of 1.6 Myr (solid red). BCAH98 models with $l_{\text {mix }}^{\text {int }}=H_{\mathrm{P}}$ for an age of $6.3 \mathrm{Myr}$ (long-dash black). BCAH98 models with $l_{\text {mix }}^{\text {int }}=1.9 \times H_{\mathrm{P}}$ for an age of $4 \mathrm{Myr}$ (dot black). The blue dots are for LkCa $3 \mathrm{~A}$ and the cyan squares for LkCa $3 \mathrm{~B}$. We adopt a distance $d=127 \mathrm{pc}$ and extinction $E(B-V)=0.10$ as in Torres et al. (2013). The numbers close to the red open circles on the solid curve give $T_{\text {eff }}$ and mass (in $M_{\odot}$ in the brackets) for a few models.

evolutionary models (relying on the NextGen atmosphere models) and the colours and magnitudes from current atmosphere models. The positions and slopes of the hybrid model isochrones are significantly different from both the new and the BCAH98 models. Using these and similar hybrid models to derive cluster ages and masses or to test them against multiple systems yields incorrect results and incorrect conclusions on the validity or invalidity of the models. Such comparisons, often seen in the literature, are totally meaningless.

\section{Conclusions}

The new set of evolutionary models presented in this paper, consistently coupling internal and atmospheric structures, show significant improvement over previous generations. They solve some of the persistent flaws present in them, such as predicting optical colours that are too blue. Significant improvement in the atmosphere models, in terms of updated molecular linelists and revised solar abundances, provide a much better match to observations. More systematic tests of current models against observations will help to identify the remaining uncertainties. The calibration of the mixing-length parameter, as presented in this work, provides overall good agreement between 1D and multi-D RHD thermal profiles. This exercise highlights the poor approximation of using a constant value for $l_{\text {mix }}^{\text {atm }}$ for pre-main sequence and main-sequence low-mass stars. The agreement, however, is not perfect, depending on the effective temperature and the surface gravity. This very likely reflects the limitation of the MLT formalism to correctly capture the thermal properties of convectively unstable atmospheres over a wide range of parameters. We note as well that remaining uncertainties in the multi-D RHD simulations are not excluded. Efforts towards developing such 

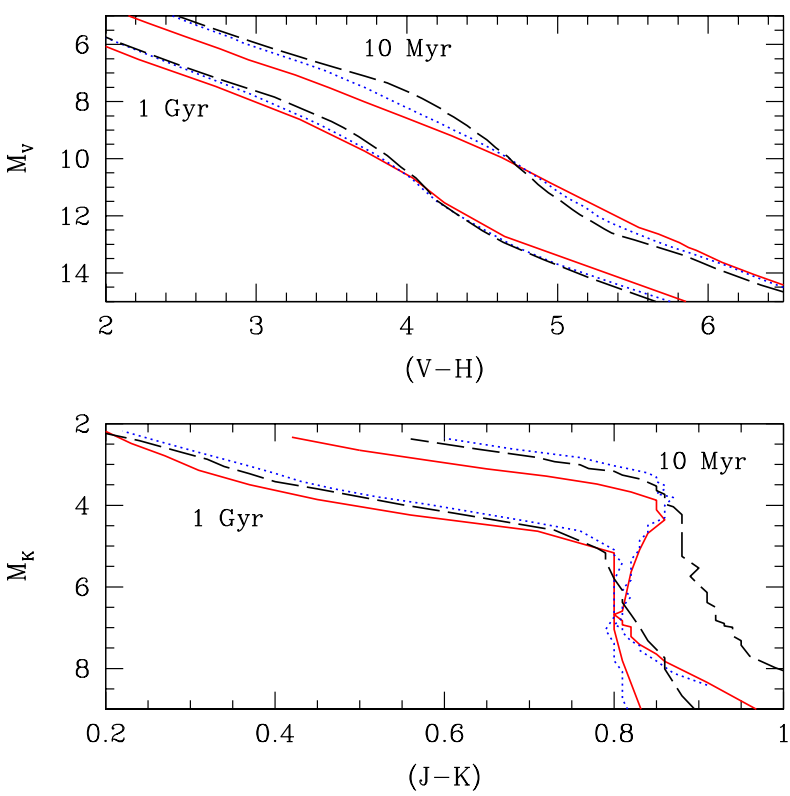

Fig. 8. Comparison of $10 \mathrm{Myr}$ and $1 \mathrm{Gyr}$ isochrones for various sets of models in two CMDs. Solid (red): present models. Long dash (black): BCAH98 models with $l_{\text {mix }}^{\text {int }}=H_{\mathrm{P}}$. Dot (blue): hybrid models using the BCAH98 structure models and the colours/magnitudes of present atmosphere models.

simulations in cool atmospheres need to be pursued. Since models at young ages are particularly sensitive to the treatment of atmospheric convection (Baraffe et al. 2002), comparisons between models and observations of objects in young clusters (age $\lesssim 10 \mathrm{Myr}$ ) will help to improve the treatment of convection.

Following the release of these new models down to the hydrogen-burning limit ${ }^{1}$, we are currently working on their extension to L, T, and Y dwarfs, including dust formation and settling. This will provide a complete and coherent set of models for low-mass stars and brown dwarfs down to Jupiter-mass objects.

Acknowledgements. This work is supported by Royal Society award WM090065. It is also partly supported by the ERC under the European Community's Seventh Framework Programme (FP7/2007-2013 Grant Agreement No. 247060), the consolidated STFC grant ST/J001627/1, the French ANR through the GuEPARD project grant ANR10-BLANC0504-01, and the PNPS of the CNRS (INSU). We thank the CHARA team (Russel White, Todd Henry, Cassy Davidson, Justin Cantrell) for providing the CHARA data and Guillermo Torres for providing information on $\mathrm{LkCa} 3$. We thank José Caballero for discussion and Richard Freedman and Jonathan Tennyson for sharing their insights into the status of molecular line data. The model atmospheres used in this study were calculated at the PSMN of the ENS-Lyon and at the GWDG in co-operation with the Institut für Astrophysik Göttingen.

\section{References}

Allard, F., Hauschildt, P. H., Alexander, D. R., Tamanai, A., \& Schweitzer, A. 2001, ApJ, 556, 357

Allard, F., Homeier, D., \& Freytag, B. 2012a, Roy. Soc. London Philos. Trans. Ser. A, 370, 2765

Allard, F., Homeier, D., Freytag, B., \& Sharp, C. M. 2012b, in EAS Pub. Ser. 57, eds. C. Reylé, C. Charbonnel, \& M. Schultheis, 3

Antia, H. M., \& Basu, S. 2011, J. Phys. Conf. Ser., 271, 012034

Asplund, M., Grevesse, N., \& Sauval, A. J. 2005, in Cosmic Abundances as Records of Stellar Evolution and Nucleosynthesis, ASP Conf. Ser., 336, 25

Asplund, M., Grevesse, N., Sauval, A. J., \& Scott, P. 2009, ARA\&A, 47, 481

Baraffe, I., Chabrier, G., Allard, F., \& Hauschildt, P. H. 1998, A\&A, 337, 403

Baraffe, I., Chabrier, G., Allard, F., \& Hauschildt, P. H. 2002, A\&A, 382, 563

Baraffe, I., Chabrier, G., Barman, T. S., Allard, F., \& Hauschildt, P. H. 2003, A\&A, 402, 701

Baraffe, I., Chabrier, G., \& Gallardo, J. 2009, ApJ, 702, L27

Baraffe, I., Vorobyov, E., \& Chabrier, G. 2012, ApJ, 756, 118

Barber, R. J., Tennyson, J., Harris, G. J., \& Tolchenov, R. N. 2006, MNRAS, 368, 1087

Basu, S., \& Antia, H. M. 2013, J. Phys. Conf. Ser., 440, 012017

Bernath, P. 2006, in Astrochemistry - From Laboratory Studies to Astronomical Observations, eds. R. I. Kaiser, P. Bernath, Y. Osamura, S. Petrie, \& A. M. Mebel, AIP Conf. Ser., 855, 143

Caffau, E., Ludwig, H.-G., Steffen, M., Freytag, B., \& Bonifacio, P. 2011, Sol. Phys., 268, 255

Cantrell, J. R., Henry, T. J., \& White, R. J. 2013, AJ, 146, 99

Chabrier, G., \& Baraffe, I. 1997, A\&A, 327, 1039

Chabrier, G., Baraffe, I., Allard, F., \& Hauschildt, P. 2000, ApJ, 542, 464

Dotter, A., Chaboyer, B., Jevremović, D., et al. 2008, ApJS, 178, 89

Freytag, B., Allard, F., Ludwig, H.-G., Homeier, D., \& Steffen, M. 2010, A\&A, 513, A19

Freytag, B., Steffen, M., Ludwig, H.-G., et al. 2012, J. Comput. Phys., 231, 919 Grevesse, N., Noels, A., \& Sauval, A. J. 1993, A\&A, 271, 587

Hauschildt, P. H., Allard, F., Ferguson, J., Baron, E., \& Alexander, D. R. 1999, ApJ, 525, 871

Hoeijmakers, H. J., de Kok, R. J., Snellen, I. A. G., et al. 2015, A\&A, 575, A20

Jorgensen, U. G. 1994, A\&A, 284, 179

Kippenhahn, R., \& Weigert, A. 1990, Stellar Structure and Evolution (Berlin, Heidelberg, New York: Springer Astron. Astrophys. Lib.)

Leggett, S. K. 1992, ApJS, 82, 351

Ludwig, H.-G., Freytag, B., \& Steffen, M. 1999, A\&A, 346, 111

Ludwig, H.-G., Allard, F., \& Hauschildt, P. H. 2002, A\&A, 395, 99

Miller, S., Tennyson, J., Jones, H. R. A., \& Longmore, A. J. 1994, in Molecules in the Stellar Environment (Berlin: Springer Verlag), ed. U. G. Jorgensen, IAU Colloq. 146, Lect. Notes Phys., 428, 296

Monet, D. G., Dahn, C. C., Vrba, F. J., et al. 1992, AJ, 103, 638

Partridge, H., \& Schwenke, D. W. 1997, J. Chem. Phys., 106, 4618

Peña Ramírez, K., Béjar, V. J. S., Zapatero Osorio, M. R., Petr-Gotzens, M. G., \& Martín, E. L. 2012, ApJ, 754, 30

Plez, B. 1998, A\&A, 337, 495

Rajpurohit, A. S., Reylé, C., Allard, F., et al. 2013, A\&A, 556, A15

Schwenke, D. W. 1998, Faraday Discussions, 109, 321

Serenelli, A. M., \& Basu, S. 2010, ApJ, 719, 865

Tashkun, S. A., Perevalov, V. I., Teffo, J.-L., et al. 2004, in SPIE Conf. Ser. 5311, eds. L. N. Sinitsa, \& S. N. Mikhailenko, 102

Torres, G., Ruíz-Rodríguez, D., Badenas, M., et al. 2013, ApJ, 773, 40

\footnotetext{
1 Isochrones are available at: http://emps.exeter.ac.uk/ physics-astronomy/staff/ib233 or http://perso.ens-lyon. fr/isabelle.baraffe/BHAC15dir
} 